\title{
Direction-of-arrival and Range Estimation of Wideband Sources in the Near Field
}

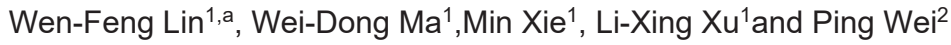 \\ ${ }^{1}$ Institute of Electronic Engineering, China Academy of Engineering Physics, Mianyang 621900, China \\ ${ }^{2}$ Department of Electronic Engineering, University of Electronic Science and Technology of China, Chengdu, Sichuan, China
}

\begin{abstract}
In this letter, we address the problem of wideband sources localization in the near field of the sensor array. It is shown that the spatial sampling rate can be considered as a function of temporal frequency so that wideband sources can be represented in the spatial frequency domain. A computationally efficient localization method is then developed based on the Toeplitz matrices constructed from the frequency-dependent cross-correlation between sensors. This allows us to resolve near field sources with quite a few numbers of sensors. Indeed, the sources can be located with fewer sensors. Finally, the simulation results are presented to validate the performance of the proposed method.
\end{abstract}

\section{Introduction}

The localization of radiating sources using sensors arrays is a problem of considerable importance, occurring in a variety of fields ranging from radar, sonar, wireless communication, and radio astronomy to seismology[1-4]. Therefore, this problem has received considerable attention in the literature, resulting in a plethora of estimation algorithms [5-7]. However, the majority of such algorithms have been restricted to DOA estimate of narrowband sources in the far-field. Recently, there has been growing interest in developing sources location estimators in the near-field, in which case, both the angle and the range are required to be estimated simultaneously. Huang and Barkat[8] presented two techniques, i.e., twodimension (2-D) MUSIC method and the maximum likelihood estimator (MLE), to determine the range and the angle of sources, which can be considered to be the modified versions of far-field cases. In [9-12], a series of ESPRIT-like methods are proposed, where higher order statistics is involved. Lately, some computationally simple second-order statistics-based methods have been proposed in the literature, such as the cyclic-based method [13], the weighted linear prediction method [14], the generalized ESPRIT method [15-16], the CRB-Based method[17-18], the NF-DSPE and the JADSSE method [19] etc. Recently, mixed near-field and far-field sources localization are proposed[20-21]. Significantly, most of the aforementioned methods rely on the narrowband assumption, therefore, to deal with wideband signals, e.g., acoustic or seismic, in [22-23] the authors provided some schemes to solve the problem of joint range and DOA estimation for multiple wideband near field sources. However, these methods have been designed under the assumptions that the number of the sources can be estimated is less than or equal to the number of antenna elements. This is a central problem in some environments and situations in which the number of sensors is limited by physical or economical considerations.

Therefore, the objective of our letter is to propose a novel scheme to simultaneously estimating the DOA and sensor location using fewer sensors, possibly less than the number of wideband sources in the near-field. Like the conventional methods for wideband sources[24-26], the received data is transformed to the frequency domain firstly, and each frequency bin can be represented by the narrowband model. Secondly, we make use of the fact that the relative distance between different elements (i.e. the physical size measured in wavelengths) is a function of the temporal frequency. Therefore, the Toeplitz matrices including the angle and the range information can be obtained by the frequency-dependent crosscorrelation between sensors[27]. Then, we can employ the traditional subspace-based methods to estimate the angle and the range of wideband near field sources. Finally, the simulation results show that our proposed technique can improve greatly the detection and estimation capability for wideband near field sources.

\section{Problem Formulation and Proposed Method}

Consider $\mathrm{K}$ near field, wideband, and uncorrelated radiating sources observed by a passive nonuniform antenna array of five sensors indicated in Figure1,

\footnotetext{
${ }^{\mathrm{a}}$ Corresponding author: linwenfeng_job@163.com
} 


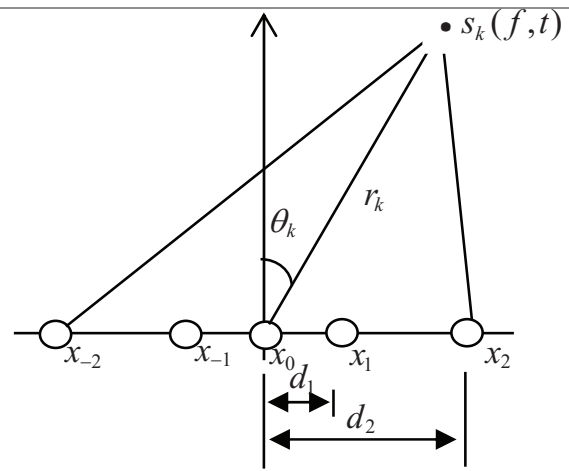

Figure 1. Near-field, wideband sources observed by a five nonuniform linear array which is symmetric about the original element.

where the array center is the origin and the phase reference point. Since we consider wideband sources in the near field, the array signal model in the frequency domain can be given by

$$
x_{p}(f)=\sum_{k=1}^{K} s_{k}(f) e^{j \tau_{p k}(f)}+N_{p}(f) \quad p=0, \pm 1, \pm 2
$$

where $s_{k}(f), N_{p}(f)$, and $\tau_{p k}(f)$ denote the $k$ th source signal spectrum, the additive noise, and the delay associated with the $k$ th source signal propagation time between the original sensor at the $p$ th sensor in the $f$ frequency band, respectively. The noise $N_{p}(f)$ is a zeromean spatially white process uncorrelated with the signal sources, and $\tau_{p k}(f) \approx \omega_{p k}(f)+\phi_{p k}(f)[14]$ and $\omega_{p k}$ and $\phi_{p k}$ are given by

$$
\begin{gathered}
\omega_{p k}(f)=\left\{\begin{array}{cc}
-p 2 \pi f d_{1} \sin \left(\theta_{k}\right) / c & p= \pm 1 \\
-p \pi f d_{2} \sin \left(\theta_{k}\right) / c & p= \pm 2 \\
0 & p=0
\end{array}\right. \\
\phi_{p k}(f)=\left\{\begin{array}{cc}
\pi f d_{1}^{2} \cos ^{2}\left(\theta_{k}\right) / c r_{k}(f) & p= \pm 1 \\
\pi f d_{2}^{2} \cos ^{2}\left(\theta_{k}\right) / c r_{k}(f) & p= \pm 2 \\
0 & p=0
\end{array}\right.
\end{gathered}
$$

where $c$ is the speed of the signal propagation in the considered medium, and $r_{k}(f)=r_{k} c / f$ is a frequency dependent value.

In addition, the wideband sources are assumed to be the same power spectrum over a given frequency band $\left[f_{\min }, f_{\max }\right]$ provided with symmetric characteristic with respect to the central frequency $f_{0}=\frac{f_{\min }+f_{\max }}{2}$, i.e., $s_{k}\left(f_{0}+n \Delta f\right)=s_{k}\left(f_{0}-n \Delta f\right) k=1, \cdots, K, n=1, \cdots, N \quad$, without loss of generality, where we can make the following assumptions that each wideband source can be decomposed into $2 N+1$ frequency bins with interfrequency bin difference $\Delta f=\frac{f_{\max }-f_{\min }}{2 N}$.

Next, making use of the assumptions mentioned above, we can define the cross-correlation between $x_{1}\left(f_{0}+n \Delta f\right)$ and $x_{-1}\left(f_{0}+n \Delta f\right)$ or $x_{2}\left(f_{0}+n \Delta f\right)$ and $x_{-2}\left(f_{0}+n \Delta f\right)$ as

$$
\begin{aligned}
& r_{p}\left(f_{0}+n \Delta f\right) \square E\left\{x_{p}\left(f_{0}+n \Delta f\right) x_{-p}^{*}\left(f_{0}+n \Delta f\right)\right\} \\
& =E\left\{\left(\sum_{k=1}^{K} s_{k}\left(f_{0}+n \Delta f\right) e^{j\left(\omega_{p k}\left(f_{0}+n \Delta f\right)+\phi_{p k}\left(f_{0}+n \Delta f\right)\right)}\right)\left(\sum_{k=1}^{K} s_{k}\left(f_{0}+n \Delta f\right) e^{j\left(-\omega_{p k}\left(f_{0}+n \Delta f\right)+\phi_{p k}\left(f_{0}+n \Delta f\right)\right)}\right)^{*}\right\} \\
& +E\left\{N_{p}\left(f_{0}+n \Delta f\right) N_{-p}^{*}\left(f_{0}+n \Delta f\right)\right\} \\
& =\sum_{k=1}^{K} P_{k}\left(f_{0}+n \Delta f\right) e^{j 2 \omega_{p k}\left(f_{0}+n \Delta f\right)} \quad p=1,2 \quad n=-N, \cdots,-1,0,1, \cdots, N
\end{aligned}
$$

where $E\{\bullet\}$ and $(\square)^{*}$ denote the statistical expectation and the complex conjugate, respectively. Additionally, $P_{k}\left(f_{0}+n \Delta f\right)=E\left\{s_{k}\left(f_{0}+n \Delta f\right) s_{k}^{*}\left(f_{0}+n \Delta f\right)\right\} \quad$ is the power spectral of the $k$ th source at frequency $f_{0}+n \Delta f$. To simplify the descriptions, we will consider the scenario as in [28] where the power spectral is constant in different frequency in the subsequent section.

Thus, the cross-correlation in (4) can be rewritten by

$$
r_{p}(n) \square r_{p}\left(f_{0}+n \Delta f\right)=\sum_{k=1}^{K} P_{k f_{0}} e^{j 2 \omega_{p k}(n \Delta f)} \square \sum_{k=1}^{K} P_{k f_{0}} e^{j n \alpha_{p k}}
$$

where $\quad P_{k f_{0}} \square P_{k} e^{j 2 \omega_{p k}\left(f_{0}\right)} \quad, \quad \alpha_{p k} \square 2 \omega_{p k}(\Delta f) \quad, \quad$ and $p=1,2 n=-N, \cdots,-1,0,1, \cdots, N$, then we can construct the following Toeplitz matrixs $\mathbf{R}_{p, 1}[27]$ and by the crosscorrelations $\left\{r_{p}(n)\right\}_{n=-N}^{N}$,

$$
\begin{aligned}
\mathbf{R}_{p, 1} & =\left[\begin{array}{lccc}
r_{p}(0) & r_{p}(1) & \cdots & r_{p}(N) \\
r_{p}(-1) & r_{p}(0) & \cdots & r_{p}(N-1) \\
\vdots & \vdots & \ddots & \vdots \\
r_{p}(-N) & r_{p}(-N+1) & \cdots & r_{p}(0)
\end{array}\right] \\
= & \mathbf{A}_{p, 1} \mathbf{D}(\mathbf{P}) \mathbf{A}_{p, 1}^{H}
\end{aligned}
$$

where $(\square)^{H}$ denotes the complex conjugate, and $\quad \mathbf{A}_{p, 1}=\left[\mathbf{a}_{p, 1}\left(\theta_{1}\right), \mathbf{a}_{p, 1}\left(\theta_{2}\right), \cdots, \mathbf{a}_{p, 1}\left(\theta_{K}\right)\right] \quad$ with $\mathbf{a}_{p, 1}\left(\theta_{k}\right)=\left[1, \exp \left(-j \alpha_{p k}\right), \cdots, \exp \left(-j N \alpha_{p k}\right)\right]^{T}$ and $\mathbf{D}(\mathbf{P}) \square \operatorname{diag}(\mathbf{P})=\operatorname{diag}\left\{P_{1 f_{0}}, P_{2 f_{0}}, \cdots, P_{K f_{0}}\right\}$.

Since $\mathbf{A}_{p, 1}$ is a $(N+1) \times K$ Vandermonde matrix [29] with full column rank when $\theta_{i} \neq \theta_{j}(i \neq j)$, the columns are linearly uncorrelated matrix. From the expression of $P_{k f_{0}} k=1,2, \cdots, K$ in (5), we can see that $P_{k f_{0}} \neq 0$ and $\mathbf{D}(\mathbf{P})$ has a rank of $K$. Therefore, the rank of $\mathbf{R}_{p, 1}$ equals to the number of wideband sources and the signal subspace or noise subspace can be obtained by singular value decomposition (SVD). Finally, the traditional narrowband subspace-based DOA techniques [30] are applicable to $\mathbf{R}_{p, 1}$ in (6) to obtain $\left\{\alpha_{p k}\right\}_{k=1}^{K}$, and the DOAs are estimated by the simple operation.

Then we also define the cross-correlation between $x_{1}\left(f_{0}+n \Delta f\right)$ and $x_{-1}\left(f_{0}-n \Delta f\right)$ or $x_{2}\left(f_{0}+n \Delta f\right)$ and $x_{-2}\left(f_{0}-n \Delta f\right)$ as 


$$
\begin{aligned}
& r_{p}(n \Delta f) \square E\left\{x_{p}\left(f_{0}+n \Delta f\right) x_{-p}^{*}\left(f_{0}-n \Delta f\right)\right\}
\end{aligned}
$$

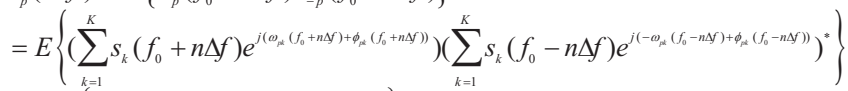

$$
\begin{aligned}
& +E\left\{N_{p}\left(f_{0}+n \Delta f\right) N_{-p}^{*}\left(f_{0}-n \Delta f\right)\right\}
\end{aligned}
$$

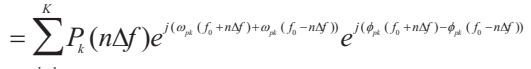

$$
\begin{aligned}
& =\sum_{k=1}^{k=1} P_{k}(n \Delta f) e^{j 2 \omega_{\mu}\left(S_{0}\right)} e^{j \pi d d_{p}^{2} \cos ^{2}\left(\theta_{k}\right) 4 f_{0} n \Delta / c^{2} c_{k}} \quad p=1,2 n=-N, \cdots,-1,0,1, \cdots, N
\end{aligned}
$$

where $P_{k}(n \Delta f) \square E\left\{s_{k}\left(f_{0}+n \Delta f\right) s_{k}^{*}\left(f_{0}-n \Delta f\right)\right\}$ $=P_{k}\left(f_{0}+n \Delta f\right)=P_{k}\left(f_{0}-n \Delta f\right)=P_{k}$, then Eq.(6) can be rewritten as

$$
r_{p \mathscr{f}}(n) \square r_{p}(n \Delta f)=\sum_{k=1}^{K} P_{k f_{0}} e^{j \pi d_{p}^{2} \cos ^{2}\left(\theta_{k}\right) 4 f_{0} n \Delta f / c^{2} r_{k}} \square \sum_{k=1}^{K} P_{k f_{0}} e^{j n \beta_{p k}}
$$

where $p=1,2, n=-N, \cdots,-1,0,1, \cdots, N$,

$$
P_{k f_{0}} \square P_{k} e^{j 2 \omega_{p k}\left(f_{0}\right)}, \beta_{p k} \square \pi d_{p}^{2} \cos ^{2}\left(\theta_{k}\right) 4 f_{0} \Delta f / c^{2} r_{k} .
$$

In the similar way to (6), we can construct the following Toeplitz matrixs $\mathbf{R}_{p, 2}$,

$$
\begin{aligned}
\mathbf{R}_{p, 2} & =\left[\begin{array}{cccc}
r_{p \Delta f}(0) & r_{p \Delta f}(1) & \cdots & r_{p \Delta f}(N) \\
r_{p \Delta f}(-1) & r_{p \Delta f}(0) & \cdots & r_{p \Delta f}(N-1) \\
\vdots & \vdots & \ddots & \\
r_{p \Delta f}(-N) & r_{p \Delta f}(-N+1) & \cdots & r_{p \Delta f}(0)
\end{array}\right] \\
= & \mathbf{A}_{p, 2} \mathbf{D}(\mathbf{P}) \mathbf{A}_{p, 2}^{H}
\end{aligned}
$$

where $\mathbf{A}_{p, 2}=\left[\mathbf{a}_{p, 2}\left(\theta_{1}, r_{1}\right), \mathbf{a}_{p, 2}\left(\theta_{2}, r_{2}\right), \cdots, \mathbf{a}_{p, 2}\left(\theta_{K}, r_{K}\right)\right]$ with $\mathbf{a}_{p, 2}\left(\theta_{k}, r_{k}\right)=\left[1, \exp \left(-j \beta_{p k}\right), \cdots, \exp \left(-j N \beta_{p k}\right)\right]^{T}$ and

$\mathbf{D}(\mathbf{P}) \square \operatorname{diag}(\mathbf{P})=\operatorname{diag}\left\{P_{1 f_{0}}, P_{2 f_{0}}, \cdots, P_{K f_{0}}\right\}$.Similarly, we can also obtain $\left\{\beta_{p k}\right\}_{k=1}^{K}$ and then the ranges of wideband sources can be estimated by

$$
r_{k}=4 \pi d_{p}^{2} \cos ^{2}\left(\theta_{k}\right) f_{0} \Delta f / c^{2} \beta_{p k} \quad k=1, \cdots, K
$$

Remark: It is worth noting that for unique estimation of the DOA and the range, we need to make the following assumption

$$
d_{p} \leq c / 2 \Delta f \text { and } d_{p} \leq c \sqrt{r_{\min } / f_{0} \Delta f} / 2
$$

where $r_{\max }$ denote the minimum value in the set $\left\{r_{k}\right\}_{k=1}^{K}$. Without loss of generality, we can employ $\mathbf{R}_{2,1}$ and $\mathbf{R}_{1,2}$ to estimate the DOA and the range respectively due to a tradeoff between the estimation performance and the phase ambiguity in the following simulations.

\section{Simulations}

We now evalutate the performance of the proposed method in estimating the DOAs and the range of wideband near-field sources. The spectral range of the wideband sources is taken to be the normalized frequency interval $[0.8,1.2]$ with respect to the central frequency $f_{0}$, that is a bandwidth of $40 \%$ of $f_{0}$, where we decompose the wideband sources into 21 frequency bins with $\Delta f=0.02$. The element spacing $d_{1}=c \sqrt{r_{\min } / f_{0} \Delta f} / 2$ and $d_{2}=c / 2 \Delta f$ is considered.

Firstly, we examine the estimation performance of the proposed method. The impinging source signals are two wideband sources signals located at $\left[\theta_{1}, r_{1}\right]=\left[-10^{\circ}, 4\right]$ and $\left[\theta_{2}, r_{2}\right]=\left[5^{0}, 2\right]$, respectively. The performance is measured by the normalized root mean square error (RNMSE) for the range and the root mean square error (RMSE) for the DOA defined in [12]. The results shown below are based on 1000 independent trials. Figure 2 and Figure3. show the RMSE and RNMSE of the estimated source angles and ranges with respect to the SNR from $-3 \mathrm{~dB}$ to $15 \mathrm{~dB}$. It is observed that the RMSE of DOA estimation is very small even at low SNR; for example, the RMSE is only 0.2 degree for $\mathrm{SNR}=-2 \mathrm{~dB}$. In addition, we also see that the smaller the range is, the better estimation performance is.

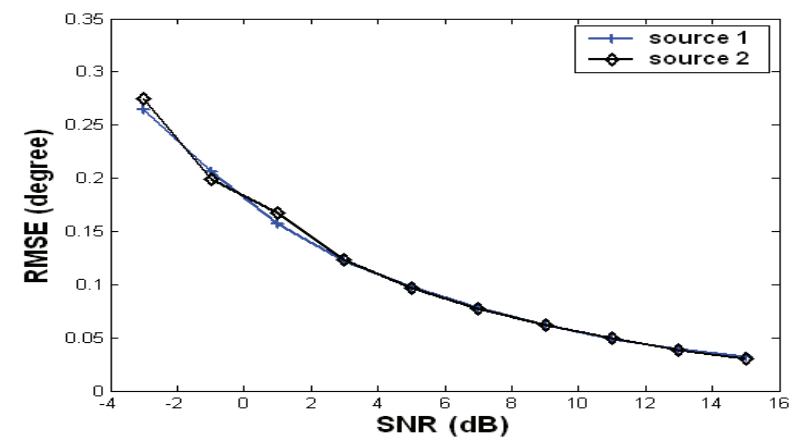

Figure 2. RMSE of the angle estimation versus SNR for two wideband near field sources.

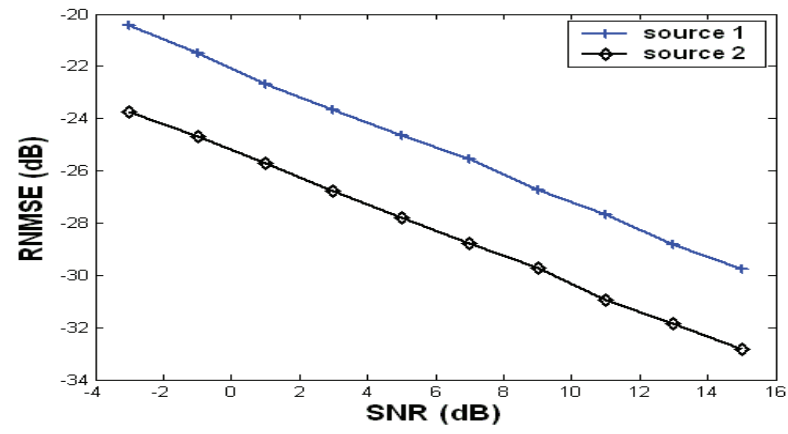

Figure 3. NRMSE of the range estimation versus SNR for two wideband near field sources.

Next, we examine the estimation capability for multiple sources of the proposed method shown Figure4(a) and (b) show that the DOA of five and six near-field uncorrelated wideband sources are placed at $\quad\left[\begin{array}{llll}-20^{\circ}-10^{\circ} & 0^{\circ} & 10^{\circ} & 20^{\circ}\end{array}\right] \quad$ and

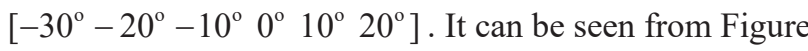
4 that we can estimate much more wideband near field sources than the number of sensors while it is impossible for the conventional methods to deal with more sources than sensors. 


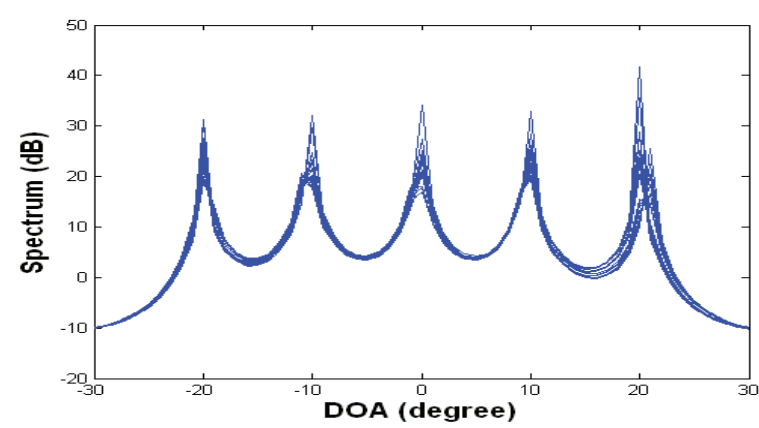

(a)

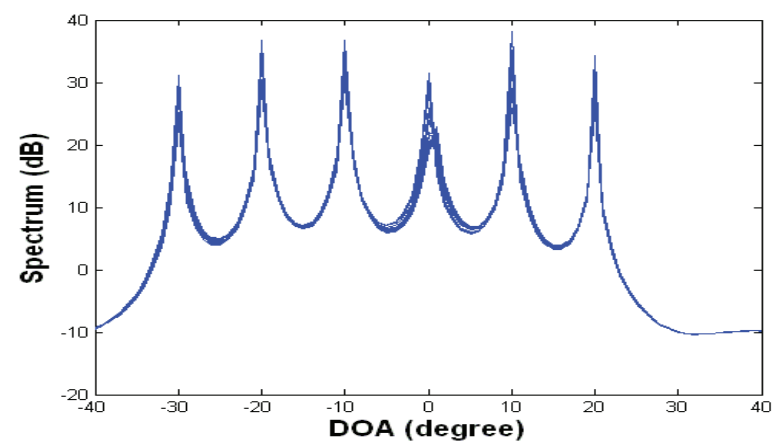

(b)

Figure 4. DOA estimation of uncorrelated wideband sources by the proposed algorithm, (a) five sources $\left[\begin{array}{llll}-20^{\circ}-10^{\circ} & 0^{\circ} 10^{\circ} 20^{\circ}\end{array}\right]$ at $\mathrm{SNR}=5 \mathrm{~dB},(\mathrm{~b})$ six sources $\left[\begin{array}{llll}-30^{\circ}-20^{\circ}-10^{\circ} & 0^{\circ} & 10^{\circ} & 20^{\circ}\end{array}\right]$ at $\mathrm{SNR}=10 \mathrm{~dB}$.

\section{Conclusions}

We have presented a novel joint DOA and range estimation algorithm for wideband near field sources to deal with the scenarios in which there are more sources than sensors. This method takes advantage of the characteristics of wideband sources and obtains crosscorrelation between sensors at each frequency bin to form Toeplitz matrixs that contains all DOA and range information. As a result, DOA and range estimation can be achieved using the conventional narrowband signal subspace techniques. Simulation results have demonstrated the effectiveness of the proposed method.

\section{References}

1. S. Haykin, Adaptive Radar Signal Processing, John Wiley \& Sons, Hoboken, NJ( 2007)

2. P. S. Naidu, Sensor Array Signal Processing, CRC Press LLC( 2001)

3. T. K. Sarkar, M. C. Wicks, M. Salazar-Palma, and R. J. Bonneau, Smart Antennas, John Wiley \& Sons, Hoboken, NJ( 2003)

4. J. Capon, "High-resolution frequency-wavenumber spectrum analysis," Proc. IEEE, vol. 57, pp. 24082418(1969)

5. J. Krim and M. Viberg, "Two decades of array signal processing research: The parametric approach,"
IEEE Signal Processing Mag., vol.13, pp. 6794( 1996)

6. L.Gan, W.F. Lin, X.Y. Luo , P. Wei, "Estimation 2D angle of arrival with a cross-correlation matrix," Journal of the Chinese Institute of Engineers, vol.36, pp.667-671(2013)

7. L.Gan, X.Y. Luo, "Direction-of-arrival estimation for uncorrelated and coherent signals in the presence of multipath propagation," IET Microw. Antennas Propag., vol.7, pp.746-753(2013)

8. Y. D. Huang and M. Barkat, "Near-field multipe source localization by passive sensor array," IEEE Trans. Antennas Propag., vol. 39, no. 7, pp. 968-975, (1991)

9. Y. Wu, L. Ma, C. Hou, G. Zhang, and J. Li, "Subspace-based method for joint range and DOA estimation of multiple near-field sources," Signal Process., vol.86, pp:2129-2133(2006)

10. R. N. Challa and S. Shamsunder, "High-order subspace-based algorithms for passive localization of near-field sources," in Proc. 29th Asilomar Conf. Signals, Syst., Comput., Pacific Grove, CA, pp. 777781 (1995)

11. M. Haardt, R. N. Challa, and S. Shamsunder, "Improved bearing and range estimation via highorder subspace based Unitary ESPRIT," in Proc. 30th Asilomar Conf. Signals, Systems, and Computers, Pacific Grove, CA, pp. 380-384 (1996)

12. Y.H. Xu, A.G. Wang, W. Leng and Z.E. Nie, "Joint Frequency, 2-D DOA and Range Estimation of Near Field Sources," Third International Conference on Measuring Technology and Mechatronics Automation, pp. 455-458(2011)

13. J-H. Lee and C-H. Tung, "Estimating the bearings of near-field cyclostationary signals," IEEE Trans. Signal process., vol. 50, pp: 110-118( 2002)

14. E. Grosicki, K. Abed-Meraim and Y. Hua, "A weighted linear prediction method for near-field source localization," IEEE Trans. Signal process., vol. 53, pp: 3651-3660(2005)

15. W. Zhi and M. Y-W Chia, "Near-field source location via symmetric subarrays," IEEE Signal Process. Lett., vol.14, pp:409-412(2007)

16. J. Xie, H.H. Tao, X. Rao and J. Su, "Efficient Method of Passive Localization for Near-Field Noncircular Sources," IEEE Antennas and Wireless Propag., vol. 14, pp. 1223-1226( 2015)

17. H. Gazzah and J.P. Delmas, "CRB-Based Design of Linear Antenna Arrays for Near-Field Source Localization," IEEE Trans. Antennas Propag., vol. 62, no. 4, pp. 1965-1974( 2014)

18. J.P. Delmas, M.N.E. Korso, H. Gazzah and M. Castella, "CRB analysis of planar antenna arrays for optimizing near-field source localization," Signal Processing, pp. 117-134(2016)

19. J.A. Chaaya, J. Picheral, and S. Marcos, "Localization of spatially distributed near-field sources with unknown angular spread shape," Signal Processing, pp. 259-265( 2015)

20. J. Xie, H.H. Tao, X. Rao and J. Su, " Localization of mixed far-field and near-field sources under 
unknown mutual coupling," Digital Signal Processing, pp. 229-239( 2016)

21. J.J. Jiang, F.H. Duan, J. Chen, Y.C. Li and X.N. Hua, "Mixed Near-Field and Far-Field Sources Localization Using the Uniform Linear Sensor Array," IEEE Seneors Journal, pp. 3136-3143(2013)

22. J. C. Chen, R. E. Hudson and K. Yao, "MaximumLikelihood source localization and unknown sensor location estimation for wideband signals in the nearfield," IEEE Trans. Signal process., vol. 50, pp: 1843-1854( 2002)

23. C. Serap, C. Erdinc, and C. Hakan Ali, "Location estimation of wideband Sources with expectation maximization algorithm," Signal Processing and Communications Applications, 2007. SIU 2007. IEEE 15th, pp:11-13( 2007)

24. M. Wax, T-J. Shan and T. Kailath, "Spatio-temporal spectral analysis by eigenstructure methods," IEEE Trans. ASSP, Vol. ASSP-32, No.4,pp:817-827(1984)

25. F. Sellone, "Robust auto-focusing wideband DOA estimation," Signal Processing, Vol. 86, pp:1737(2006)

26. Y-S Yoon, L. M. Kaplan and J. H. McClellan, "TOPS: New DOA estimator for wideband signals," IEEE Trans. Signal Process., Vol.54, pp:1977-1989 (2006)

27. J.-F. Gu, N.-J. Li, P. Wei and H.-M. Tai, "Wideband DOA estimation using two sensors," 2010 International Conference on Measuring Technology and Mechatronics Automation, pp:1095-1098( 2010)

28. S. Valaee and P. Kabal, "Estimation of directions of arrival of wideband and wideband spread sources," Signal Processing, Vol. 87, pp:614-622( 2007)

29. C. D. Meyer, "Matrix Analysis and Applied Linear Algebra", SIAM(2000)

30. A. J. van der Veen, E.F. Deprettere, and A.L. Swindlehurst, "Subspace-based signal analysis using singular value decomposition," Proc. IEEE, vol. 81, pp. 1277-1308(1993) 